\title{
Modificações do consumo de cigarros e bebidas alcoólicas em adolescentes de Curitiba, Paraná: um estudo longitudinal
}

\author{
Changes in tobacco and alcoholic beverage consumption among \\ adolescents from Curitiba in the State of Paraná: a longitudinal \\ study
}

Michael Pereira da Silva (https://orcid.org/0000-0002-7628-3997) ${ }^{1}$
Edmar Roberto Fantineli (https://orcid.org/0000-0001-9536-359X) ${ }^{2}$
Eliane Denise Araújo Bacil (https://orcid.org/0000-0002-8672-395X) ${ }^{2}$
Thiago Silva Piola (https://orcid.org/0000-0002-6081-0510) ${ }^{2}$
Nicolau Augusto Malta Neto (https://orcid.org/0000-0003-4385-4287) ${ }^{2}$
Wagner de Campos (https://orcid.org/0000-0003-3979-1017) ${ }^{3}$

${ }^{1}$ Grupo de Pesquisa em Atividade Física e Saúde Pública, Faculdade de Medicina, Universidade Federal de Rio Grande. Rua Visconde de Paranaguá 102 sala 411, Centro. 96200190 Rio Grande RS Brasil. michael.ufpr@hotmail.com ${ }^{2}$ Centro de Estudos em Atividade Física e Saúde, Universidade Federal do Paraná. Curitiba PR Brasil.

${ }^{3}$ Departamento de Educação Física, Universidade Federal do Paraná. Curitiba PR Brasil.

\begin{abstract}
The scope of this study was to verify alterations in cigarette and alcohol consumption in a sample of adolescents from the state education system of the city of Curitiba in the State of Parana between 2015 and 2017. It involved a longitudinal prospective cohort study conducted with 799 adolescents (11 to 15 years of age) between 2015 and 2017. The Youth Risk Behavior Surveillance System (YRBS) questionnaire evaluated mild and excessive alcohol and tobacco consumption. Modifications of alcohol and tobacco consumption were evaluated through Generalizable Estimating Equation (GEE) models with Poisson distribution to obtain Incidence Risk Ratios (IRR) with 95\% confidence intervals $(95 \% \mathrm{CI})$. The "time" variable was treated as an independent variable along with other possible predictors (gender, age bracket, nutritional status, socioeconomic level, head of household education, physical activity and screen time). Tobacco consumption (IRR $=1,32,95 \% \mathrm{CI}$ $=1,02 ; 1,71)$, mild $(\operatorname{IRR}=1,32,95 \% C I=1,13$; $1,53)$ and excessive $(I R R=1,21,95 \% C I=1,04$; 1,40) alcohol consumption increased between 2015 and 2017. This study indicates that adolescents participating in this research are modifying their behaviors towards a less healthy lifestyle. Key words Risk behavior, Tobacco, Binge drinking, Adolescents
\end{abstract}

Resumo A presente investigação objetivou verificar modificações no consumo de cigarro e álcool em uma amostra de adolescentes do sistema estadual de ensino da cidade de Curitiba, Paraná, Brasil entre 2015 e 2017. Estudo longitudinal de coorte prospectivo realizado com 799 adolescentes, entre 11 e 15 anos. O consumo leve e excessivo de álcool e de cigarro foram avaliados através do questionário Youth Risk Behavior Survey (YRBS). Modificações do consumo de álcool e cigarro foram avaliadas através de modelos de Equação de Estimação Generalizável com distribuição de Poisson para obtenção de razões de taxas de incidência (RTI) com intervalos de confiança de 95\% (IC95\%). A variável "tempo" foi tratada como variável independente, juntamente com demais possiveis preditores (sexo, faixa etária, estado nutricional, nivel socioeconômico, escolaridade do chefe da família, atividade física e tempo em tela). O consumo de cigarros $(R T I=1,32, I C 95 \%=1,02$; $1,71)$, consumo leve $(R T I=1,32, I C 95 \%=1,13$; $1,53)$ e excessivo de álcool $(R T I=1,21, I C 95 \%=$ $1,04 ; 1,40)$ cresceram significativamente entre 2015 e 2017. O presente estudo denota que os adolescentes participantes desta investigação estão modificando seus comportamentos em direção a um estilo de vida menos saudável.

Palavras-chave Comportamento de risco, Tabaco, Bebedeira, Adolescentes 


\section{Introdução}

Diversos estudos têm apontado o período da adolescência como uma importante fase de modificações de comportamentos relacionados à saúde, bem como, a aquisição de comportamentos de risco que permanecerão na idade adul$\mathrm{ta}^{1-7}$. Neste período da vida, fatores biológicos e psicossociais parecem favorecer a modificação de determinados comportamentos por parte do adolescente, em que modificações decorrentes da puberdade e a busca pela inserção em um grupo social parece ser um favorecedor da adoção de determinados comportamentos, muitos deles de risco a saúde s,9. $^{8,9}$.

O consumo de cigarros e de bebidas alcoólicas por parte dos adolescentes apresenta-se como importantes fatores de risco à saúde e apresentam prevalências preocupantes nessa fase da vida $^{10,11}$. Estudos com adolescentes brasileiros apontam uma variabilidade nas prevalências desses comportamentos de risco, com variação de $4,7 \%$ (Região Nordeste) a 7,6\% (Região Sul) para o consumo de cigarros e de 14,0\% (Rio Grande do Norte) a 34,0\% (Rio Grande do Sul) para o consumo de bebidas alcoólicas ${ }^{10-13}$.

A identificação de como esses comportamentos relacionados à saúde se modificam ao longo da adolescência, bem como os fatores associados a esta modificação torna-se importante para que intervenções sejam estruturadas. No entanto, grande parte dos estudos envolvendo adolescentes brasileiros avaliaram estes comportamentos e seus fatores associados através de estudos transversais o que impossibilita, assim, a verificação de fatores determinantes bem como, as magnitudes de modificações destes comportamentos ao longo da adolescência ${ }^{14,15}$.

Evidências demonstram a elevação do consumo dessas substâncias ao longo da adolescência ${ }^{1-7}$. Contudo, estudos longitudinais envolvendo adolescentes brasileiros são escassos, principalmente no que se refere às modificações do consumo de cigarros nesta fase. Já para o consumo de bebidas alcoólicas, encontra-se, publicado recentemente, um único estudo longitudinal com adolescentes do Sudeste brasileiro (Minas Gerais), o qual verificou uma elevação na prevalência do consumo de cigarros em 4,3\% entre os anos de 2010 e $2012^{6}$.

Diante disso, a presente investigação objetivou verificar possíveis modificações no consumo de cigarro e álcool em uma amostra de adolescentes matriculados no sistema estadual de ensino da cidade de Curitiba, Paraná, entre os anos de 2015 e 2017. Adicionalmente, fatores associados a cada comportamento bem como às possíveis modificações destes comportamentos foram analisados.

\section{Materiais e Métodos}

\section{Delineamento do estudo}

Este é um estudo longitudinal de coorte prospectivo, o qual possibilitou um acompanhamento, durante um período de dois anos, de adolescentes matriculados na rede pública de ensino da cidade de Curitiba, Paraná.

\section{População e amostra}

A população foi composta por adolescentes (12,0 a 15,9 anos no momento do recrutamento dos sujeitos), de ambos os sexos, matriculados na rede pública estadual de ensino da cidade de Curitiba, Paraná. O cálculo amostral para prevalência foi realizado para a obtenção do número mínimo necessário para estimativas de prevalência do consumo de cigarros e de álcool, adotando prevalência de $50 \%{ }^{16}$, intervalo de confiança de $95 \%$, erro padrão de $5 \%$, efeito de delineamento de 1,5 e acréscimo de $30 \%$ para perdas e recusas. Utilizando-se do valor apresentado pelo Censo Escolar de Educação Básica $2015^{17}$ (124.764 alunos), a amostra necessária para a estimativas de prevalência foi de 750 participantes.

A amostra foi obtida de forma estratificada por conglomerados dentro de cada uma das nove regionais pertencentes ao Núcleo Regional de Educação de Curitiba no ano de 2015 (Bairro Novo, Boa Vista, Boqueirão, Cajuru, CIC, Matriz, Pinheirinho, Portão, Santa Felicidade) favorecendo a seleção de escolas situadas em regiões com características socioeconômicas distintas da cidade de Curitiba, Paraná (Figura 1) Para cada regional de ensino, duas escolas foram selecionadas aleatoriamente, com exceção de uma regional (Bairro Novo), que obteve apenas uma escola sorteada. No total, 17 escolas foram sorteadas e convidadas a participarem do estudo, no entanto, três escolas: Boqueirão (1), CIC (1) e Santa Felicidade (1) não aceitaram participar do estudo.

Cada escola forneceu de 4 a 6 turmas do sexto ano do Ensino Fundamental ao primeiro ano do Ensino Médio que foram intencionalmente selecionadas de acordo com as possibilidades de logística da equipe pedagógica das instituições de ensino, as quais ofereceram turmas, não possuí- 
Figura 1. Regionais administrativas de Curitiba, Paraná e respectivas rendas médias por domicílio.

Fonte: IPPUC - Instituto de Pesquisa e Planejamento Urbano de Curitiba, 2016 (https://www.ippuc.org.br/).

am avaliações, e que tinham aulas de Educação Física nos dias agendados para a coleta de dados. Todos os alunos pertencentes às turmas selecionadas foram convidados a participarem do estudo. Sendo assim, foram coletados dados de 799 adolescentes que estavam dentro da faixa etária estipulada para a inclusão na coorte (12 a 15,9 anos). A Figura 2 apresenta o número de participantes válidos obtidos em cada fase do estudo para cada regional e serie.

\section{Instrumentos e procedimentos}

\section{Coleta de dados}

As coletas de dados tiveram início no mês de agosto de 2015. As escolas sorteadas foram contatadas e informadas sobre o conteúdo e objetivos do estudo e o aceite da escola ocorreu mediante assinatura do diretor concordando com a realização dos procedimentos do estudo em sua instituição nas três fases do projeto: Baseline (2015), Fase 2 (2016) e Fase 3 (2017).

Os adolescentes foram convidados a participar do estudo com dois dias de antecedência ao dia agendado para coleta de dados. Duas cópias do termo de consentimento livre e esclarecido (TCLE) e do termo de assentimento informado livre e esclarecido (TALE) foram disponibilizadas. A participação dos adolescentes no estudo foi condicionada à assinatura do TCLE por pais ou responsável legal e à assinatura do TALE pelo adolescente.

Todos os procedimentos de coleta de dados foram realizados dentro do ambiente da escola/ colégio. Os adolescentes foram convidados a preencher questionários sobre informações sociodemográficas, atividade física, comportamento sedentário, consumo de álcool, consumo de cigarro e foram submetidos à avaliação de peso $\mathrm{e}$ estatura, bem como a autoavaliação da maturação sexual. Todos os procedimentos realizados apresentaram duração média de 80 minutos e foram realizados por uma equipe treinada de membros e colaboradores do Centro de Estudos em Atividade Física e Saúde da Universidade Federal do Paraná. O presente estudo foi autorizado pela Secretaria de Estado e Educação do Estado do Paraná e aprovado pelo Comitê de Ética em 


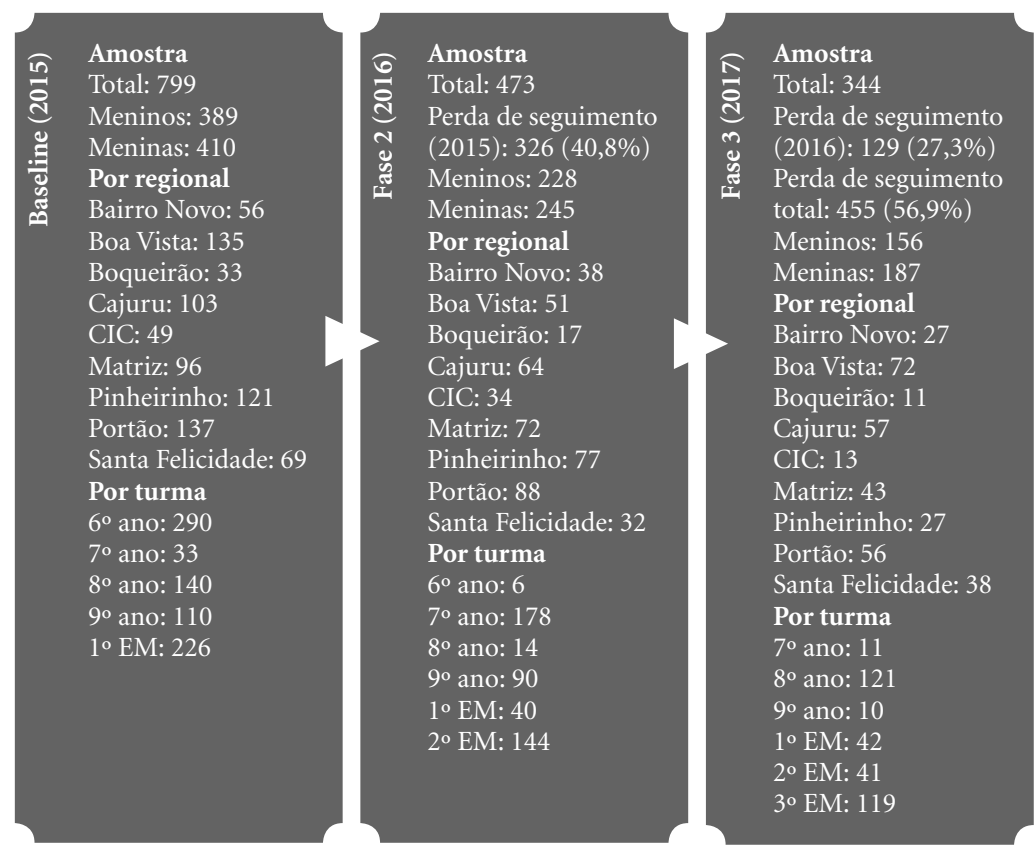

Figura 2. Amostra e perda de seguimento entre 2015 e 2017.

Fonte: Elaborado pelos autores.

Pesquisa com seres humanos da Universidade Federal do Paraná.

\section{Consumo de cigarros e bebida alcoólica dos adolescentes}

Para a identificação do consumo de cigarros e bebida alcoólica nos adolescentes foram utilizadas questões da versão em português do Youth Risk Behavior Survey (YRBS) ${ }^{18}$. As questões referentes ao consumo de cigarros e de bebidas alcoólicas abordaram aspectos referentes a frequência e quantidade de consumo destas substâncias nos 30 dias que antecederam a coleta de dados. Adolescentes que reportaram consumo de um ou mais cigarros nos últimos 30 dias foram classificados com a presença do comportamento de risco. Adicionalmente, para o consumo de álcool, duas questões avaliaram o consumo leve e o consumo excessivo de álcool, respectivamente. Com relação à identificação do consumo leve, este foi definido como consumir ao menos uma dose em pelo menos um dia dos últimos 30 dias. Já para a ocorrência do consumo excessivo, que trata do consumo de cinco doses ou mais de bebidas alcoólicas em uma mesma ocasião, aqueles ado- lescentes que apresentaram este comportamento em pelo menos um dia dos últimos 30 dias foram classificados com o comportamento de risco ${ }^{19}$.

\section{Covariáveis}

\section{Classificação econômica e escolaridade do chefe da família}

A classe econômica foi avaliada mediante aplicação do questionário de Critério de Classificação Econômica Brasil proposto pela Associação Brasileira de Empresas de Pesquisa ${ }^{20}$. O presente instrumento estima uma faixa de renda familiar média e proporcionou que os participantes fossem classificados em sete classes: A, B1, B2, C1, C2 e D-E.

A questão sobre escolaridade do chefe da família presente neste questionário também foi utilizada de forma isolada como possível preditor do consumo de cigarros e álcool. Esta questão oferece opções de resposta que vão do "Analfabeto/Fundamental I incompleto" ao "Superior Completo".

Para fins de análise, esta variável foi dividida em três categorias: a) Baixo: envolvendo os níveis de escolaridade de Analfabeto até Fundamental 
II incompleto); b) Médio: envolvendo os níveis de escolaridade de Fundamental completo até Superior incompleto e; c) Alto: envolvendo aqueles com no mínimo ensino Superior completo.

\section{Estado nutricional}

O estado nutricional foi avaliado através do cálculo do índice de massa corporal (IMC) obtido através da razão entre massa corporal (kg) e a estatura ( $\mathrm{m}$ ) elevada ao quadrado. A avaliação da massa corporal foi realizada mediante utilização de uma balança antropométrica digital portátil (W721, Wiso, Brasil) com resolução de 100 gramas e capacidade de 150 quilogramas. A identificação do estado nutricional foi realizada mediante as classificações dos escores $\mathrm{z}$ de IMC para cada sexo e idade propostos pela Organização Mundial da Saúde: Baixo peso $=<-2 \mathrm{DP}, \mathrm{Eu}-$ trófico $\geq-2 \mathrm{DP}$ e $<+1 \mathrm{DP}$, Sobrepeso $=\geq+1 \mathrm{DP}$ $\mathrm{e}<+2 \mathrm{DP}$ e Obesidade $=\geq+2 \mathrm{DP}^{21}$.

\section{Participação em atividade física}

O questionário Youth Activity Profile (YAP) foi utilizado para avaliar os níveis de participação em atividade física (AF). O YAP é um questionário autoadministrado recordatório de sete dias desenvolvido para medir a atividade física em crianças e adolescentes. Ele é composto por 10 itens de múltipla escolha que perguntam sobre a AF realizada em diferentes contextos (dentro da escola, fora da escola e final de semana). Todas as questões foram pontuadas em um intervalo de 1 a 5 e pontuações mais elevadas representaram níveis mais altos de participação em AF. As associações de YAP com atividade física moderada a vigorosa intensidade (AFMV) medida objetivamente foram positivas e moderadas $(\mathrm{p}<0,001)^{22}$. O YAP foi adaptado e validado com adolescentes brasileiros de 11 a 17 anos de idade apresentando correlação moderada (rho: 0,44; p <0,001) entre os escores do YAP e a AFMV medida por acelerometria ${ }^{23}$. Os adolescentes foram divididos em tercis (baixa, média e alta participação em AF) baseado nos tercis escores totais de participação em atividade física.

\section{Tempo em atividade sedentária}

O tempo em atividade sedentária foi avaliado através do Questionário de Atividades Sedentárias para Adolescentes (QASA) ${ }^{24}$ o qual fornece informações sobre o tempo gasto em diferentes tipos de atividades sedentárias, tanto em final quanto em dias de semana, de uma semana típica. O QASA apresenta indicadores positivos de validade e reprodutibilidade para a avaliação do tem- po sedentário em adolescentes brasileiros $(\mathrm{CCI}=$ 0,88 (IC95\% 0,82-0,91); rho =0,79, p<0,01 $)^{24,25}$. Para as análises nesse estudo, utilizamos o tempo em tela (Televisão, Vídeo games, DVDs e computadores). Adolescentes que apresentaram $\geq 4$ horas de tela por dia foram classificados com alto comportamento sedentário.

\section{Tratamento dos dados e análise estatística}

Análise descritiva e de perda de seguimento

Para a descrição da amostra, utilizou-se a análise descritiva para a identificação de medidas de tendência central e dispersão para as variáveis contínuas, e distribuição de frequência para variáveis categóricas. Para verificar as possíveis diferenças entre os adolescentes que apresentaram dados nas três fases do estudo e àqueles que apenas apresentaram dados na primeira fase do estudo (Fase 1-Baseline), foram utilizados Testes $t$ independentes para as variáveis contínuas e teste de qui-quadrado para as variáveis categóricas.

\section{Modificações e fatores associados ao consumo de cigarros e bebidas alcoólicas}

Para a verificação das modificações e fatores associados ao consumo de cigarro, consumo de álcool e consumo excessivo de álcool, utilizou-se modelos de Equação de Estimação Generalizável adotando distribuição de Poisson para obtenção de razões de taxas de incidência (RTI) com intervalos de confiança de 95\% (IC95\%). Pesos amostrais foram utilizados em todas as análises levando em consideração a probabilidade de seleção da escola em cada regional e a probabilidade da seleção da turma e dos alunos dentro de cada escola. Adicionalmente, utilizamos modelos de correção dos erros padrões das estimativas de regressão baseados em controles de erros robustos por conglomerado.

Para cada comportamento analisado, a variável "tempo" - 2015, 2016 e 2017- foi tratada como variável independente juntamente com a inserção de uma série de demais possíveis variáveis explicativas tais como: sexo, faixa etária, estado nutricional, nível socioeconômico, nível educacional do chefe da família, nível de participação em atividades físicas e tempo em tela. $O$ consumo de cigarros, o consumo leve de álcool e o consumo excessivo de álcool nos 30 dias que antecederam a coleta de dados foram utilizados como variáveis dependentes nos modelos de regressão.

As variáveis presentes em cada modelo de predição foram inseridas de forma forçada nos modelos iniciais e retiradas sucessivamente 
caso não apresentassem significância estatística $(\mathrm{p}<0,05)$.

Todas as análises de regressão foram realizadas utilizando o pacote $x t$ de modelos de análise longitudinal do software estatístico Stata 14.1 MP com nível de significância estatística estabelecido em $\mathrm{p}<0,05$. Taxas de incidência foram calculadas cada modelo de regressão mediante o comando de pós estimação predict.

\section{Resultados}

\section{Descrição da amostra}

No total, 455 adolescentes não apresentaram dados das três fases do estudo. Os principais motivos para as perdas foram: a) mudança do adolescente para outra escola; b) evasão escolar e; c) ausência do adolescente nas datas agendadas para a coleta de dados. Uma maior proporção de meninos (51,5\% vs. $\left.44,1 \%, \chi^{2}=4,30, \mathrm{p}=0,04\right)$, de consumo de cigarros $\left(11,8 \%\right.$ vs. $6,6 \%, \chi^{2}=5,87$, $\mathrm{p}=0,01)$ e de consumo excessivo de bebidas alcoólicas $\left(19,7 \%\right.$ versus $10,7 \%, \chi^{2}=11,40, p<$ $0,01)$ foi verificado no grupo de adolescentes que não apresentaram dados nas 3 fases do estudo em comparação com aqueles com dados válidos em todas as fases.

A Tabela 1 apresenta características comportamentais da amostra para cada ano de acompanhamento. Aproximadamente $42 \%$ dos adolescentes apresentaram-se classificados com sobrepeso/obesidade. Adolescentes eram em sua maioria de baixo nível socioeconômico (NSE) $(50,8 \%)$, com o chefe da família de baixo nível educacional $(52,2 \%)$. Com relação ao consumo de cigarros e bebidas alcoólicas, 9,8\% dos adolescentes reportaram terem consumido cigarro, $24,2 \%$ reportaram consumo leve de álcool e $16,1 \%$ reportaram consumo excessivo de álcool.

\section{Consumo de cigarros e álcool}

A Tabela 2 apresenta as razões de taxa de incidência para as modificações e fatores associados ao consumo de cigarro, consumo leve e excessivo de álcool nos 30 dias que antecederam a coleta de dados. Com relação ao consumo de cigarros ao menos uma vez nos últimos 30 dias que antecederam à coleta de dados, verificou-se que a ocorrência desse comportamento foi 32\% maior para cada ano de acompanhamento $(\mathrm{RTI}=1,32$, IC95\% $=1,02 ; 1,71, p=0,032)$. Tais resultados podem ser melhor visualizados na Figura 3 , que apresenta as elevações nas taxas de incidência de consumo de cigarros, consumo leve e excessivo de álcool entre 2015 e 2017. Como fatores associados a este comportamento verificou-se que a ocorrência do consumo de cigarros foi maior nos adolescentes mais velhos (RTI=1,98, IC95\% = $1,31 ; 2,99, \mathrm{p}<0,001)$ e com sobrepeso ou obesidade $(\mathrm{RTI}=1,69, \mathrm{IC} 95 \%=1,34 ; 2,13, \mathrm{p}=0,001)$.

O consumo leve de álcool (RTI= 1,32, IC95\% $=1,13 ; 1,53, \mathrm{p}<0,001)$ e o consumo excessivo de álcool $(\mathrm{RTI}=1,21$, IC95\% = 1,04; 1,40, p=0,009) nos 30 dias que antecederam à coleta de dados para cada um dos três anos de seguimento também apresentaram crescimentos significativos entre 2015 e 2017. Mais especificamente, a ocorrência de consumo leve e do consumo excessivo foi $32 \%$ e $21 \%$ maior para cada ano de acompanhamento, respectivamente. (Figura 3). Meninas $(\mathrm{RTI}=1,48, \mathrm{IC} 95 \%=1,22 ; 1,78, \mathrm{p}=0,001)$, adolescentes mais velhos ( RTI $=2,45$, IC95\% = $2,02 ; 2,97, \mathrm{p}<0,001)$ e adolescentes com níveis mais altos de AF (RTI= 1,13, IC95\% = 1,04; 1,22, $\mathrm{p}=0,002$ ) apresentaram maior ocorrência do consumo leve álcool nos 30 dias que antecederam a coleta de dados. Já para o consumo excessivo de álcool nos 30 dias que antecederam à coleta de dados, meninas, adolescentes mais velhos, com sobrepeso e obesidade e alto nível socioeconômico apresentaram maior ocorrência desse comportamento com RTI variando de 1,42 (Sexo; IC95\%= 1,15; 1,75, p=0,001) a 2,88 (Faixa etária; IC95\%= 2,29; 3,62, p >0,001). Por outro lado, adolescentes com chefe da família com maior nível educacional apresentaram menor ocorrência do consumo excessivo de álcool nos 30 dias que antecederam à coleta de dados em cada um dos três anos de seguimento (RTI= $0,83$, IC $95 \%=0,70 ; 0,99, \mathrm{p}=0,043)$.

\section{Discussão}

O consumo de cigarros e bebidas alcoólicas entre adolescentes ainda representa um grande problema de saúde pública no mundo $0^{6,7,26,27}$. Ambas as substâncias se associam com a adoção de demais comportamentos de risco, bem como a morbidade e a mortalidade precoce decorrentes de doenças crônico-degenerativas, e seu monitoramento ao longo da adolescência é de suma importância para a identificação de padrões de comportamento, eficiência de políticas voltadas ao combate ao uso bem como, criação de novas estratégias de prevenção a adoção destes comportamentos de risco $^{26,28-30}$. 
Tabela 1. Características sociodemográficas e comportamentais dos adolescentes de Curitiba,Paraná em cada fase do estudo $(2015,2016,2017)$.

\begin{tabular}{|c|c|c|c|}
\hline & $2015(n=799)$ & $2016(n=427)$ & $2017(n=315)$ \\
\hline & Média (DP) & Média (DP) & Média (DP) \\
\hline Idade & $13,6(1,6)$ & $14,7(1,9)$ & $15,7(1,8)$ \\
\hline \multicolumn{4}{|l|}{ Escores de Atividade física } \\
\hline Total & $2,36(0,71)$ & $2,24(0,71)$ & $2,20(0,72)$ \\
\hline \multicolumn{4}{|l|}{ Comportamento sedentário (min/dia) } \\
\hline \multirow[t]{2}{*}{ Tempo em tela } & $233,0(148,2)$ & $204,0(125,9)$ & $180,8(139,8)$ \\
\hline & $\%$ & $\%$ & $\%$ \\
\hline \multicolumn{4}{|l|}{ Sexo } \\
\hline Meninos & $48,7 \%$ & $48,2 \%$ & $45,5 \%$ \\
\hline Meninas & $51,3 \%$ & $51,8 \%$ & $54,5 \%$ \\
\hline \multicolumn{4}{|l|}{ Maturação sexual } \\
\hline Pré-púbere & $2,4 \%$ & $1,9 \%$ & $0,3 \%$ \\
\hline Púbere & $83,9 \%$ & $72,3 \%$ & $58,8 \%$ \\
\hline Pós-púbere & $13,7 \%$ & $25,8 \%$ & $40,9 \%$ \\
\hline \multicolumn{4}{|l|}{ Estado Nutricional } \\
\hline Normal & $58,4 \%$ & $57,6 \%$ & $59,0 \%$ \\
\hline Sobrepeso/Obesidade & $41,6 \%$ & $42,4 \%$ & $41,0 \%$ \\
\hline \multicolumn{4}{|l|}{ Nível socioeconômico } \\
\hline Baixo (C,D e E) & $50,8 \%$ & $50,1 \%$ & $47,3 \%$ \\
\hline Alto (A e B) & $49,2 \%$ & $49,9 \%$ & $52,7 \%$ \\
\hline \multicolumn{4}{|l|}{ Nível educacional do chefe da família } \\
\hline Baixo & $52,2 \%$ & $52,6 \%$ & $54,2 \%$ \\
\hline Médio & $28,1 \%$ & $28,3 \%$ & $28,1 \%$ \\
\hline Alto & $19,7 \%$ & $19,1 \%$ & $17,7 \%$ \\
\hline \multicolumn{4}{|l|}{ Consumo de Cigarro } \\
\hline Não & $90,2 \%$ & $85,0 \%$ & $87,2 \%$ \\
\hline$\geq 1$ cigarro nos últimos 30 dias & $9,8 \%$ & $15,0 \%$ & $12,8 \%$ \\
\hline \multicolumn{4}{|l|}{ Consumo de álcool } \\
\hline Não & $75,8 \%$ & $69,1 \%$ & $61,0 \%$ \\
\hline$\geq 1$ dose nos últimos 30 dias & $24,2 \%$ & $30,9 \%$ & $39,0 \%$ \\
\hline \multicolumn{4}{|l|}{ Consumo excessivo de álcool } \\
\hline Não & $83,9 \%$ & $78,4 \%$ & $76,2 \%$ \\
\hline$\geq 5$ doses ao menos uma vez nos últimos 30 dias & $16,1 \%$ & $21,6 \%$ & $23,8 \%$ \\
\hline
\end{tabular}

Fonte: Elaborado pelos autores.

Com relação ao consumo de cigarros, estudos apresentam-se inconsistentes em relação as modificações deste comportamento ao longo da adolescência. É possível verificar elevações na prevalência de consumo de cigarros com taxas variando entre $9,1 \%$ a $24,0 \%{ }^{1,2,31}$. Por outro lado, estabilidade ${ }^{32}$ e até mesmo reduções no consumo de cigarro foram visualizados com taxas de prevalência do não uso de cigarros crescendo em aproximadamente $20 \%{ }^{33}$. Os dados do presente estudo demonstram a elevação da ocorrência do consumo de cigarros com crescimento de aproximadamente $7,0 \%$ entre 2015 e 2017.
De forma similar ao consumo de cigarros, a ocorrência do consumo leve de álcool $(\geq 1$ vez nos últimos 30 dias que antecederam à coleta de dados) e do consumo excessivo ( $\geq 5$ doses em uma única ocasião nos últimos 30 dias que antecederam a coleta de dados) cresceu entre 2015 e 2017. A ocorrência desses comportamentos cresceu aproximadamente $13 \%$ para o consumo leve e $4 \%$ para o consumo excessivo. A elevação do consumo de álcool na adolescência é bem reportada na literatura, com aumento elevações nas prevalências de consumo entre $4,3 \%{ }^{6}$ a $44,3 \%{ }^{7}$. Dados mais preocupantes são apresentados por 
um estudo de acompanhamento de adolescentes dos 10 aos 15 anos de idade em que a probabili- dade de consumo de álcool cresceu aproximadamente $50 \%$.

Tabela 2. Razão de taxas de incidência para o consumo de cigarro, consumo leve e excessivo de álcool em adolescentes de Curitiba, Paraná de 2015 a 2017.

\begin{tabular}{|c|c|c|c|c|c|c|}
\hline & \multicolumn{2}{|c|}{$\begin{array}{c}\text { Consumo de cigarros } \\
\text { ( } \geq 1 \text { cigarro nos últimos } \\
30 \text { dias })\end{array}$} & \multicolumn{2}{|c|}{$\begin{array}{c}\text { Consumo leve de álcool } \\
(\geq 1 \text { dose nos últimos } 30 \\
\text { dias })\end{array}$} & \multicolumn{2}{|c|}{$\begin{array}{c}\text { Consumo excessivo de álcool } \\
\text { ( } \geq 5 \text { doses em ao menos } 1 \text { dia } \\
\text { nos últimos } 30 \text { dias) }\end{array}$} \\
\hline & $\begin{array}{c}\text { RTI } \\
(\text { IC95\%) }\end{array}$ & p & $\begin{array}{c}\text { RTI } \\
\text { (IC95\%) } \\
\end{array}$ & $\mathbf{p}$ & $\begin{array}{c}\text { RTI } \\
(\text { IC95\%) }\end{array}$ & $\mathbf{p}$ \\
\hline Tempo & $\begin{array}{c}1,32 \\
(1,02 ; 1,71)\end{array}$ & 0,032 & $\begin{array}{c}1,31 \\
(1,13 ; 1,53)\end{array}$ & $<0,001$ & $\begin{array}{c}1,21 \\
(1,04 ; 1,40)\end{array}$ & 0,009 \\
\hline Sexo & - & - & $\begin{array}{c}1,48 \\
(1,22 ; 1,78)\end{array}$ & 0,001 & $\begin{array}{c}1,42 \\
(1,15 ; 1,75)\end{array}$ & 0,001 \\
\hline Faixa etária (2015) & $\begin{array}{c}1,98 \\
(1,32 ; 2,13)\end{array}$ & $<0,001$ & $\begin{array}{c}2,45 \\
(2,02 ; 2,97)\end{array}$ & $<0,001$ & $\begin{array}{c}2,88 \\
(2,29 ; 3,62)\end{array}$ & $<0,001$ \\
\hline $\begin{array}{l}\text { Estado nutricional } \\
\text { (2015) }\end{array}$ & $\begin{array}{c}1,69 \\
(1,34 ; 2,13)\end{array}$ & 0,001 & - & - & $\begin{array}{c}1,42 \\
(1,05 ; 1,92)\end{array}$ & 0,022 \\
\hline $\begin{array}{l}\text { Nível socioeconômico } \\
\text { (2015) }\end{array}$ & - & - & - & - & $\begin{array}{c}1,56 \\
(1,21 ; 2,01)\end{array}$ & 0,001 \\
\hline $\begin{array}{l}\text { Nível educacional do } \\
\text { chefe da família }\end{array}$ & - & - & - & - & $\begin{array}{c}0,83 \\
(0,70 ; 0,99) \\
\end{array}$ & 0,043 \\
\hline $\begin{array}{l}\text { RTI: Razão de taxas de inci } \\
\text { feminino; Faixa etária }(201 \\
\text { Nível socioeconômico: } 0= \\
\text { (Analfabeto a Fundamenta }\end{array}$ & Baixo (Classes & e E); $1=$ & (Classes A e & Nível edu & $\begin{array}{l}1 ; 2=2017 ; \text { Sexc } \\
\text { o Normal; } 1=\text { So } \\
\text { ional do chefe d }\end{array}$ & $\begin{array}{l}\text { nasculino; } 1= \\
\text { o/Obesidade } \\
\text { lia: } 0=\text { Baixo } \\
\text { lto (Superior }\end{array}$ \\
\hline
\end{tabular}

Fonte: Elaborado pelos autores.

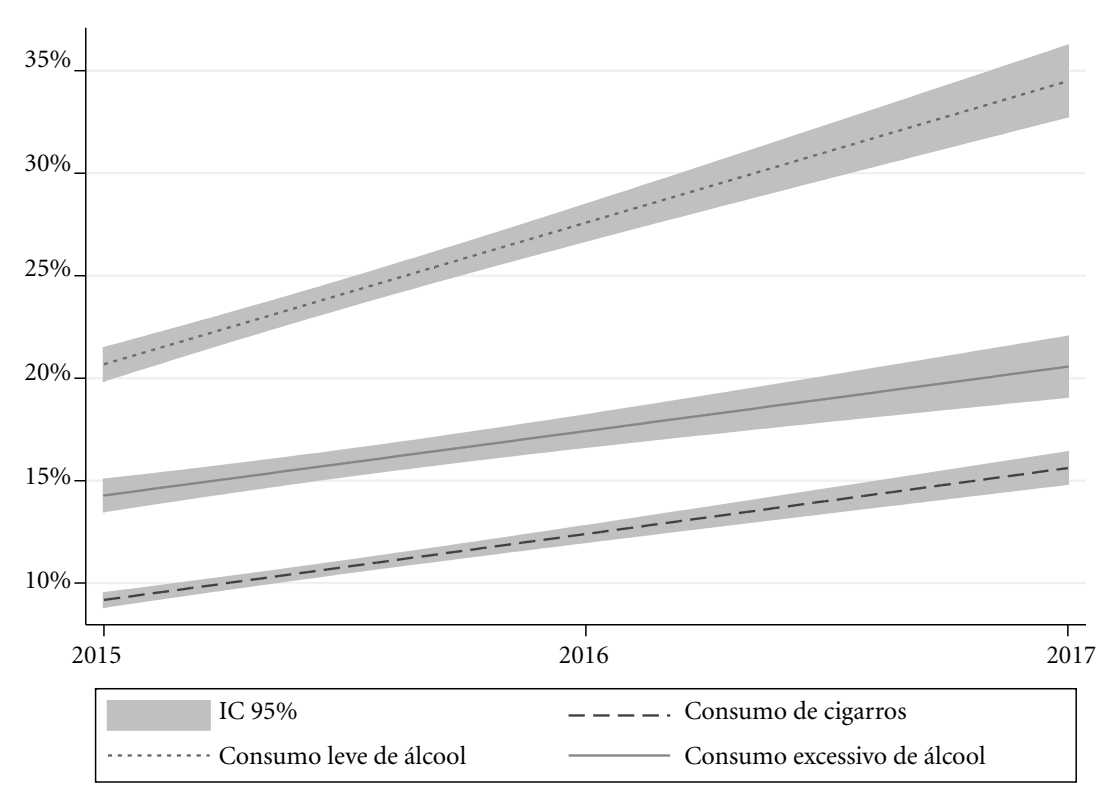

Figura 3. Incidência e intervalo de confiança de $95 \%$ de consumo de cigarros e álcool em adolescentes de Curitiba, Paraná entre 2015 e 2017. 
$\mathrm{Na}$ presente investigação, o sexo feminino apresentou-se associado a ocorrência do consumo leve e excessivo de álcool nos 30 dias anteriores a coleta dos dados em cada um dos 3 anos de seguimento, mais especificamente, meninas apresentaram taxas de incidência 48\% (consumo leve) e $42 \%$ (consumo excessivo) maiores que os meninos. Tais resultados divergem de estudos que não evidenciaram diferença entre os sexos ${ }^{6}$ ou uma menor propensão do sexo feminino a esses comportamentos de risco ${ }^{34-38}$. Os resultados do presente estudo fornecem importantes informações sobre o direcionamento de intervenções voltadas à redução do consumo destas substâncias indicando o sexo feminino como importante alvo de intervenções voltadas a este propósito.

Verificou-se também que a ocorrência do consumo de cigarros, consumo leve e excessivo de álcool nos 30 dias anteriores à coleta dos dados em cada um dos três anos de seguimento foi cerca de duas vezes maior nos adolescentes mais velhos ( $\geq 14$ anos) do que adolescentes mais novos mais especificamente, as RTI variaram de 1,98 a 2,88. A literatura apresenta-se consistente ao indicar que adolescentes mais velhos, mais especificamente aqueles com idade $\geq 15$ anos, são mais susceptíveis a apresentarem o consumo destas substâncias ${ }^{6,35,37,39-43}$. Com o avanço da idade, o adolescente está cada vez mais vulnerável à influência dos grupos sociais em que se insere, bem como a busca de uma imagem de adulto independente. Conflitos familiares são comuns nesta fase da vida e os pais exercem menor influência como moduladores do comportamento de seus filhos. Tais fatores são de suma importância para a experimentação e o consumo regular tanto de drogas lícitas quanto de ilícitas ${ }^{8,9,32}$.

O sobrepeso e obesidade associou-se diretamente com os consumos de cigarro e consumo excessivo de álcool nos 30 dias anteriores à coleta dos dados em cada um dos três anos de seguimento do presente estudo. Como descrito acima, o consumo de cigarro e álcool na adolescência sofre grande influência do meio social em que o adolescente está inserido. Este consumo baseiase na necessidade de busca por uma determinada aprovação neste meio social ou mesmo na busca de alívio ou solução para conflitos característicos desta fase da vida. Adolescentes com sobrepeso e obesidade são mais susceptíveis a condições de isolamento social por parte de seus pares, e tal isolamento pode agravar as consequências sociais e emocionais decorrentes desta condição ${ }^{42,44}$. O maior consumo dessas substâncias observados nos adolescentes do presente estudo pode ser re- flexo de estratégias de inserção e aceitação em determinados grupos sociais ou até mesmo estratégias compensatórias para enfrentar os problemas relacionados à marginalização desta condição por parte da sociedade em que se inserem, no entanto, o presente estudo não coletou informações necessárias para confirmar essa suposição. Adicionalmente, o uso de cigarro e bebidas alcoólicas por indivíduos com sobrepeso e obesidade reflete uma situação preocupante de agregação de múltiplos fatores de risco à saúde que tendem a permanecer na idade adulta e, desta forma, elevar o risco de morbidade e mortalidade ${ }^{28,45}$.

Alguns estudos têm apontado características socioeconômicas como favorecedoras do consumo de álcool ${ }^{35,36}$. No presente estudo, adolescentes com NSE médio e alto foram mais propensos ao consumo excessivo de álcool nos 30 dias anteriores à coleta dos dados em cada um dos três anos de seguimento do estudo. Tais associações indicam que o maior poder econômico parece favorecer a compra destas substâncias com maior frequência, bem como a possibilidade de frequentar bares e clubes noturnos os quais parecem favorecer maior consumo destas substâncias $^{35}$. Adicionalmente, Mattos et al. ${ }^{35}$ verificaram que aqueles adolescentes que trabalham foram mais propensos a consumirem álcool com frequência ( $R P=1,26$, IC95\% = 1,04; 1,53), no entanto, o presente estudo não apresenta dados sobre esta variável.

A literatura apresenta a atividade física e o tempo sedentário como importantes fatores moduladores de saúde em indivíduos de diversas faixas etárias. O primeiro comportamento demonstra-se muitas vezes associado a condições benéficas à saúde, enquanto o segundo é classificado como importante fator de risco. No presente estudo, a incidência das formas de consumo de álcool avaliadas foram maiores nos adolescentes com alto nível de atividade física mas não naqueles com alto tempo em tela. Com relação à associação da atividade física com o consumo dessas substâncias, a literatura apresenta resultados conflitantes. Estudos de caráter transversal indicam que adolescentes mais ativos tendem a consumir menos bebidas alcoólicas ${ }^{46,47}$, no entanto, outros estudos indicam que adolescentes mais ativos apresentaram maior consumo de álcool nos 30 dias anteriores à coleta dos dados em cada um dos três anos de seguimento ${ }^{48,49}$. Já para o comportamento sedentário, a falta de associação entre atividade física e consumo de álcool verificada na presente pesquisa contradizem os dados consistentes da literatura que, por sua vez, aponta 
que adolescentes com maior engajamento nesses comportamentos sedentários são mais propensos aos comportamentos de risco avaliados na presente investigação ${ }^{47-50}$.

O presente estudo demonstrou que o alto nível educacional do chefe da família foi um importante preditor de um menor consumo de álcool nos 30 dias anteriores à coleta dos dados em cada um dos três anos de seguimento, o que é corroborado em estudos transversais demonstrando que adolescente com chefes de família com mais alto nível educacional são menos propensos a consumir bebidas alcoólicas. Tal fator pode ser reflexo de um ambiente familiar mais estável e melhor diálogo entre os pais e o adolescente no intuito de prevenir o consumo de drogas lícitas ${ }^{51}$.

\section{Limitações do estudo}

O presente estudo apresenta limitações e pontos fortes que devem ser ressaltados. Inicialmente, o delineamento longitudinal adotado na presente investigação proporcionou a identificação de modificações nos comportamentos relacionados à saúde em adolescentes da cidade de Curitiba, Paraná. No entanto, como todo delineamento longitudinal, a desistência de participantes no decorrer do estudo é característica normal e pode limitar as resoluções dos objetivos propostos.

A taxa de retenção obtida na presente investigação foi de apenas $43,0 \%$ da amostra inicial e, visando melhor as características e o impacto destas perdas no presente estudo, diferenças entre os participantes que apresentaram dados completos nos três anos de acompanhamento e àqueles que em algum momento (Fases 2 e/ou 3) não forneceram seus dados foram analisadas. Esta análise identificou que as desistências não foram consideradas aleatórias visto que os participantes que desistiram do estudo foram mais propensos a serem do sexo masculino e a consumirem cigarro e álcool em excesso. Tais fatores podem ter implicado nas associações visualizadas para o sexo bem como em taxas de incidência superestimadas do consumo de cigarros e consumo excessivo de álcool.

Esta investigação utilizou uma amostra de adolescentes matriculados de 14 escolas estaduais. Escolas estas que foram selecionadas aleatoriamente dentro de nove regionais de ensino da cidade de Curitiba, Paraná, no entanto, as turmas e consequentemente os adolescentes foram selecionados de forma intencional dentro de cada escola, o que não garante a representatividade dos adolescentes de Curitiba. Adicionalmente, o calendário escolar do ano foi muito comprometido devido a uma greve nas instituições de ensino estaduais do Paraná. Tal fato dificultou o processo de amostragem impossibilitando o sorteio aleatório das turmas dentro de cada escola, bem como o número de turmas selecionadas dentro de cada escola.

Contudo, independente dessas limitações, o presente estudo conseguiu incluir adolescentes de diversas classes sociais e residentes em diferentes bairros de Curitiba.

Nesta investigação, a definição do consumo excessivo de álcool ( 5 ou mais doses em um único episódio), pode subestimar esse consumo excessivo visto que outras definições para o que é excessivo indicam uma dosagem menor para as meninas (4 ou mais doses em um único episódio), no entanto, devido à versão brasileira do questionário do YRBS, utilizamos a definição proposta pelo Centers of Disease Control ${ }^{18}$. Adicionalmente, devido ao modelo dos instrumentos utilizados para avaliar os comportamentos de uso de cigarro e álcool, os quais fornecem apenas informações do consumo realizados nos 30 dias anteriores à coleta de dados, impossibilitando a identificação de comportamentos anteriores a esse espaço temporal. Diante disso, as estimativas de incidências realizadas podem sofrer com esse viés de informação sugerindo que estudos futuros utilizem instrumentos que possibilitem uma avaliação mais precisa desses comportamentos.

É claro na literatura que a utilização de questionários para a avaliação de comportamentos em adolescentes apresenta limitações quanto à precisão do atributo avaliado. As questões do questionário utilizado para a avaliação do consumo de cigarros e álcool questiona somente a ocorrência desse comportamento nos últimos 30 dias anteriores ao preenchimento dele, o que pode limitar um melhor entendimento dos comportamentos que precederam a esse período de tempo. Adicionalmente, o instrumento utilizado para a avaliação do comportamento sedentário não contemplou de forma direta o tempo gasto no uso de celulares e smartphones na posição sentada, o que configura como uma importante limitação para a avaliação do comportamento sedentário. No entanto, os instrumentos utilizados no presente estudo apresentaram validade adequada e forneceram informações sobre o tipo e o contexto das atividades realizadas, considerado um ponto forte da presente investigação.

Por fim, este estudo não contemplou a avaliação de demais variáveis que podem influenciar 
o consumo de cigarro e álcool por adolescentes, como por exemplo, o consumo dos pais, irmãos e amigos, além da opção religiosa e estado civil dos pais, variáveis essas que podem ser importantes parta o entendimento desse contexto de uso de drogas lícitas por adolescentes.

\section{Conclusões}

Os resultados do presente estudo apontam que os adolescentes participantes desta investigação modificam seus comportamentos em direção a um estilo de vida menos saudável. $\mathrm{O}$ consumo de cigarros e álcool cresceu significativamente ao longo dos três anos de seguimento do estudo.
A faixa etária e o estado nutricional foram preditores do consumo de cigarros nos 30 dias anteriores à coleta dos dados em cada um dos três anos de seguimento do estudo. O sexo, a faixa etária e a atividade física foram preditores do consumo leve de álcool nos 30 dias anteriores à coleta dos dados em cada um dos três anos de seguimento do estudo. O sexo, a faixa etária, o estado nutricional e o nível socioeconômico foram preditores do consumo excessivo de álcool nos 30 dias anteriores à coleta dos dados em cada um dos três anos de seguimento do estudo. Por outro lado, adolescentes que possuíam em sua casa pais/ responsáveis com maior nível educacional foram menos propensos ao consumo excessivo de álcool nos 30 dias anteriores a coleta dos dados em cada um dos três anos de seguimento do estudo.

\section{Colaboradores}

MP Silva se responsabilizou pelo delineamento, análise, interpretação dos dados e redação do artigo, aprovação da versão a ser publicada. ER Fantineli fez o delineamento, análise, interpretação dos dados e redação do artigo. EA Bacil, TS Piola, NAM Neto responsabilizaram-se pela redação do artigo e revisão crítica. TS Piola, redação do artigo e revisão crítica. NAM Neto, redação do artigo e revisão crítica. W de Campos, por fim, responsabilizou-se pela revisão crítica e aprovação da versão a ser publicada. 


\section{Referências}

1. Pennanen M, Haukkala A, De Vries H, Vartiainen E. Longitudinal study of relations between school achievement and smoking behavior among secondary school students in Finland: Results of the ESFA study. Subst Use Misuse 2011; 46(5):569-579.

2. Daw J, Margolis R, Wright L. Emerging adulthood, emergent health lifestyles: sociodemographic determinants of trajectories of smoking, binge drinking, obesity, and sedentary behavior. J Health Soc Behav 2017; 58(2):181-197.

3. Salonna F, Kážmér L, Csémy L, Vašíčková J, Hublet A, Stock C. Trends in tobacco smoking among czech school-aged children from 1994 to 2014. Cent Eur J Public Health 2017; 25(Supl. 1):S42-S46.

4. Zehe J, Colder C. A latent growth curve analysis of alcohol-use specific parenting and adolescent alcohol use. Addict Behav 2014; 39(12):1701-1705.

5. Colder CR, O'Connor RM, Read JP, Eiden RD, Lengua LJ, Hawk LW, Wieczorek WF. Growth trajectories of alcohol information processing and associations with escalation of drinking in early adolescence. Psychol Addict Behav 2014; 28(3):659-670.

6. Jorge KO, Ferreira RC, Ferreira EF, Vale MP, Kawachi I, Zarzar PM. Binge drinking and associated factors among adolescents in a city in southeastern Brazil: a longitudinal study. Cad Saude Publica [Internet]. 2017 [acessado 2018 Jul 01]; 33(2): e00183115. Disponível em: https://www.scielo.br/scielo.php?script=sci_abstract\&pid=S0102-311X2017000205008\&lng=en\&nrm=iso

7. Aiken A, Clare PJ, Wadolowski M, Hutchinson D, Najman JM, Slade T, Bruno R, McBride N, Kypri K, Richard P, Mattick RP. Age of alcohol initiation and progression to binge drinking in adolescence: a prospective cohort study. Alcohol Clin Exp Res 2017:1-11.

8. Cavalcante MBPT, Alves MDS, Barroso MGT. Adolescência, álcool e drogas Promoção da Saúde. Esc Anna Nery Rev Enferm 2008;12(3):555-559.

9. Steinberg L. Neuroscience perspective on adolescent risk taking. Dev Rev 2008; 28(1):1-27.

10. Coutinho ESF, França-Santos D, Magliano ES, Bloch KV, Barufaldi LA, Cunha CF, Vasconcellos MTL, Szlko M. ERICA: Patterns of alcohol consumption in Brazilian adolescents. Rev Saúde Pública. 2016; 50(supl 1):1s-9s.

11. Barbosa Filho VC, Campos W De, Lopes ADS. Prevalence of alcohol and tobacco use among Brazilian adolescents : a systematic review. Rev Saude Publica 2012; 46(5):901-917.

12. Instituto Brasileiro de Geografia e Estatística (IBGE). Pesquisa Nacional de Saúde do Escolar 2015. Brasília: IBGE; 2016. p.132.

13. Figueiredo VC, Szklo AS, Costa LC, Kuschnir MCC, Silva TLN, Bloch KV, Szlko M. ERICA: Smoking prevalence in Brazilian adolescents. Rev Saúde Pública. 2016;50(supl 1):1s-10s.

14. Mazzardo O, Silva MP, Guimarães RF, Martins RV, Watanabe PI, Campos W. Health risk behaviors in teenagers according to gender, age and socioeconomic status. Med 2016; 49(4):321-330.
15. Legnani E, Legnani RFS, Dellagrana RA, Silva MP, Barbosa Filho VC, Campos W. Comportamentos de risco à saúde e excesso de peso corporal em escolares de Toledo, Paraná, Brasil. Motricidade 2012; 8(3):5970.

16. Luiz RR, Magnanini MMF. A lógica da determinação do tamanho da amostra em investigações epidemiológicas. Cad Saude Colet 2000; 8(2):9-28.

17. Instituto Nacional de Estudos e Pesquisas Educacionais Anísio Teixeira (Inep). Censo Escolar de Educação Básica 2013. Brasília: Inep; 2013.[acessado 2018 jun 20]. Disponível em: http://portal.inep.gov.br/basicacenso.

18. Guedes DP, Lopes CC. Validação da versão brasileira do Youth Risk Behavior Survey Validation of the Brazilian version of the 2007 Youth Risk Behavior Survey. Rev Saude Publica 2010; 44(5):840-850.

19. Kann L, Kinchen S, Shanklin SL, et al. Youth risk behavior surveillance-United States, 2013. MMWR Supl. 2014; 63(4):1-168.

20. Associação Brasileira de Empresas de Pesquisa (ABEP). Critério de Classificação Econômica Brasil. São Paulo: ABEP; 2014. [acessado 2018 jun 20]. http://www.abep.org/new/Servicos/DownloadCodigoConduta.aspx?id=02.

21. Onis M, Onyango AW, Borghi E, Siyam A, Nishida C, Siekmann J. Development of a WHO growth reference for school-aged children and adolescents. Bull World Health Organ 2007; 85(10):812-819.

22. Saint-Maurice PF, Welk GJ. Validity and calibration of the youth activity profile. Watz H, ed. PLoS One 2015; 10(12):e0143949.

23. Silva MP, Saint-Maurice PF, Piola TS, Neto NAM, Campos W. A versão brasileira do Youth Activity Profile: evidências preliminares de validade em adolescentes brasileiros. Rev Bras Atividade Física Saúde 2017; 22(S1):SS472.

24. Guimarães RDF, Silva MP, Legnani E, Mazzardo O, Campos W. Reprodutibilidade de questionário de atividades sedentárias para adolescentes brasileiros. Rev Bras Cineantropometria Desempenho Hum 2013; 15(3):276-285.

25. Bacil EDA, WatanabeE PI, Silva MP, Fantinelli ER, Bozza R, Campos W. Validade de um questionário de comportamento sedentário em escolares de 9 a 15 anos de idade. Rev Bras Cien Saude 2018; 22(4):341348.

26. World Health Organization (WHO). Global status report on noncommunicable diseases 2014. Geneva: WHO; 2014.

27. Lenk KM, Erickson DJ, Forster JL. Trajectories of Cigarette Smoking From Teens to Young Adulthood. Am J Heal Promot 2017; 0(0):0890117117696358.

28. Weitzman M, Cook S, Auinger P, Florin TA, Daniels S, Nguyen M, Winickoff JP. Tobacco smoke exposure is associated with the metabolic syndrome in adolescents. Circulation 2005; 112(6):862-869.

29. McCambridge J, McAlaney J, Rowe R. Adult consequences of late adolescent alcohol consumption: a systematic review of cohort studies. PLoS Med 2011; 8(2):e1000413. 
30. Ronksley PE, Brien SE, Turner BJ, Mukamal KJ, Ghali W. Association of alcohol consumption with selected cardiovascular disease outcomes: a systematic review and meta-analysis. BMJ 2011; 342(D671):1-13.

31. Jaber R, Taleb ZB, Bahelah R, Madhivanan P, Maziak W. Perception, intention and attempts to quit smoking among Jordanian adolescents from the Irbid Longitudinal Study. Int J Tuberc lung Dis 2016; 20(12):1689-1694.

32. Huang GC, Soto D, Fujimoto K, Valente TW. The interplay of friendship networks and social networking sites: Longitudinal analysis of selection and influence effects on adolescent smoking and alcohol use. Am J Public Health 2014; 104(8):51-59.

33. McKelvey K, Halpern-Felsher B. Adolescent cigarette smoking perceptions and behavior: tobacco control gains and gaps amidst the rapidly expanding tobacco products market from 2001 to 2015. J Adolesc Health 2017; 60(2):226-228

34. Strauch ES, Pinheiro RT, Silva RA, Horta BL. Alcohol use among adolescents: a population-based study. Rev Saude Publica 2009; 43(4):647-655.

35. Matos AM, Carvalho RC, Costa MCO, Gomes KEPS, Santos LM. Consumo frequente de bebidas alcoólicas por adolescentes escolares: estudo de fatores associados. Rev Bras Epidemiol 2010;13(2):302-313.

36. Gowing LR, Ali RL, Allsop S, Marsden J, Turf EE, West R, Witton J. Global statistics on addictive behaviours: 2014 status report. Addiction 2015; 110(6):904-919.

37. Niño MD, Cai T, Mota-Back X, Comeau J. Gender differences in trajectories of alcohol use from ages 13 to 33 across Latina/o ethnic groups. Drug Alcohol Depend 2017; 180(August):113-120.

38. Colder CR, Shyhalla K, Frndak SE. Early alcohol use with parental permission: Psychosocial characteristics and drinking in late adolescence. Addict Behav 2018; 76:82-87.

39. Horta BL, Calheiros P, Pinheiro RT, Tomasi E, Amaral KC. Tabagismo em adolescentes de área urbana na região Sul do Brasil. Rev Saude Publica 2001; 35(2):159164.

40. Horta RL, Horta BL, Pinheiro RT, Morales B, Strey MN. Tabaco, álcool e outras drogas entre adolescentes em Pelotas, Rio Grande do Sul, Brasil: uma perspectiva de gênero. Cad Saude Publica 2007; 23(4):775-783.

41. Silva MAM, Rivera IR, Carvalho ACC, Júnior ADHG, Moreira TCA. The prevalence of and variables associated with smoking in children and adolescents in the Maceió education system. J Pediatr (Rio J) 2006; 82(5):365-370

42. Cable N, Roman Mella MF, Kelly Y. What could keep young people away from alcohol and cigarettes? Findings from the UK Household Longitudinal Study. BMC Public Health 2017; 17(1):1-6.

43. Elinder LS, Heinemans N, Zeebari Z, Patterson E. Longitudinal changes in health behaviours and body weight among Swedish school children - associations with age, gender and parental education - the SCIP school cohort. BMC Public Health 2014; 14(1):640.
44. Strauss RS, Pollack HA. Social Marginalization of Overweight Children. Arch Pediatr Adolesc Med 2003;157(8):746.

45. Berlin I. Smoking-induced metabolic disorders: A review. Diabetes Metab 2008; 34(4):307-314

46. Charilaou M, Karekla M, Constantinou M, Price S. Relationship between physical activity and type of smoking behavior among adolescents and young adults in Cyprus. Nicotine Tob Res 2009; 11(8):969976.

47. Lesjak V, Stanojević-Jerković O. Physical activity, sedentary behavior and substance use among adolescents in slovenian urban area. Slov J Public Health 2015; 54(3):168-174.

48. Lebron C, Stoutenberg M, Janowsky M, Asfour L, Huang S, Prado G. The role of physical activity and sedentary behavior in substance use and risky sex behaviors in hispanic adolescents. J Early Adolesc 2017; 37(7):910-924

49. Peltzer K. Leisure time physical activity and sedentary behavior and substance use among in-school adolescents in eight african countries. Int J Behav Med 2010 17(4):271-278.

50. Dias PJP, Domingos IP, Ferreira MG, Muraro AP, Sichieri R, Gonçalves-Silva RMV. Prevalence and factors associated with sedentary behavior in adolescents. Rev Saude Publica 2014; 48(2):266-274.

51. Acosta LD, Fernández AR, Pillon SC. Factores sociales para el uso de alcohol en adolescentes y jóvenes. Rev Lat Am Enfermagem 2011; 19(spe):771-781.
Artigo apresentado em 02/07/2018

Aprovado em 27/08/2019

Versão final apresentada em 29/08/2019

Editores-chefes: Romeu Gomes, Antônio Augusto Moura da Silva 
